Aceptado para publicación:

Beléndez, M. (2018). Estrés en madres y padres de niños/as con diabetes: Validación de la versión española de la escala Problem Areas in Diabetes (PAID-PR). Ansiedad \& Estrés, 24(2-3): 136-139. https://doi.org/10.1016/j.anyes.2018.06.001.

\title{
ESTRÉS EN MADRES Y PADRES DE NIÑOS/AS CON DIABETES: \\ VALIDACIÓN DE LA VERSIÓN ESPAÑOLA DE LA ESCALA PROBLEM AREAS IN DIABETES (PAID-PR)
}

\section{RESUMEN}

La experiencia continuada de estrés asociada al cuidado de un hijo/a con diabetes puede afectar el bienestar emocional de sus progenitores. El objetivo de este estudio fue el análisis de la estructura factorial, fiabilidad y validez de la versión española de la escala PAID-PR (Problem Areas in Diabetes-Parent Revised). Un total de 237 progenitores (146 madres y 91 padres) de niños/as y adolescentes con diabetes tipo 1 (2-17 años) completaron la escala PAID-PR. El análisis factorial exploratorio identificó dos factores. Las puntuaciones en la escala total y por factores de la PAID-PR correlacionaron con las medidas de ansiedad-rasgo. Además, las madres presentaron niveles más altos de estrés. Los resultados indican que la versión española de la escala PAID-PR es una medida breve, fiable y válida que puede usarse en contextos clínicos y de investigación.

Palabras clave: diabetes tipo 1, padre, madre, estrés psicológico, validez, fiabilidad. 


\begin{abstract}
The constant experience of stress associated with caring for a child with diabetes can affect the emotional well-being of their parents. The main purpose of the present study was the analysis about the factor structure, reliability and validity of the Spanish version of the PAID-PR (Problem Areas in Diabetes-Parent Revised). 237 parents (146 mothers and 91 fathers) of children and adolescents with type 1 diabetes completed the PAIDPR. Exploratory Factor Analysis yielded two factors. As expected, PAID-PR scores correlated with measures of trait-anxiety. Mothers reported higher stress levels. Results indicate that the Spanish version of the PAID-PR is a reliable and valid brief measure useful in research and clinical settings.
\end{abstract}

Key words: diabetes tipo 1, father, mother, psychological stress, validity, reliability. 


\section{INTRODUCCIÓN}

El diagnóstico de una enfermedad crónica en un/a hijo/a constituye una fuente de estrés considerable para sus progenitores (Pinquart, 2018). Tras una progresiva adaptación a la nueva situación, su vida cotidiana presenta eventos estresantes específicos derivados de los cuidados que requiere cada enfermedad (Cousino \& Hazen, 2013). En España, la diabetes tipo 1 constituye un trastorno crónico frecuente en la infancia y la adolescencia (Barreiro et al., 2014). El cuidado de la diabetes tipo 1 implica la realización de distintas tareas varias veces al día como la administración de insulina, la medición de la glucemia, el seguimiento de un plan de comidas, la realización de una actividad física regular o el manejo de posibles hipoglucemias. Así, la naturaleza compleja del tratamiento junto a las preocupaciones por las hipoglucemias o por las complicaciones de la enfermedad pueden resultar fuentes de estrés para muchos progenitores (Hessler, Fisher, Polonsky, \& Johnson, 2016; Whittemore, Jaser, Chao, Jang, \& Grey, 2012). Esta carga del cuidado, que continúa recayendo en mayor medida en las madres explicaría, en parte, sus niveles más elevados de estrés en comparación con los padres (Haugstvedt, Wentzel-Larsen, Rokne, \& Graue, 2011; Nieuwesteeg et al., 2017).

La escala PAID (Problem Areas in Diabetes) es la medida más utilizada a nivel internacional para evaluar el estrés asociado a vivir con la diabetes en población adulta (Polonsky et al, 1995). La escala se compone de 20 ítems que se refieren a aspectos problemáticos de la enfermedad como las preocupaciones por las hipoglucemias, por la posibilidad de complicaciones futuras o el sentimiento de "quemarse" por el esfuerzo que supone el tratamiento, entre otros. La investigación sobre la validez de la escala en distintas lenguas se ha caracterizado por la ausencia de acuerdo en cuanto a su estructura factorial. Así, se han propuesto soluciones de uno a cuatro factores. En el 
caso de la versión española se obtuvo una estructura de cuatro factores (Beléndez, Hernández-Mijares, Marco, Domínguez, \& Pomares, 2014), similar a la encontrada con muestras de Holanda o Estados Unidos (Snoek, Pouwer, Welch, \& Polonsky, 2000).

Esta escala ha sido adaptada y, recientemente revisada (PAID-PR), para su uso con progenitores de niños/as y adolescentes con diabetes (Markowitz et al., 2012). La PAID-PR contiene 12 ítems adaptados de la escala original y 6 nuevos específicos. Los análisis psicométricos de la versión original identificaron dos factores, cargas cotidianas y preocupaciones por el futuro. Niveles más altos de estrés se asociaron con mayor frecuencia de conflicto familiar relacionado con la diabetes y menor calidad de vida de sus hijos/as según los progenitores. Sin embargo, las puntuaciones en la PAID-PR no se relacionaron ni con edad de sus hijos/as ni con la duración de la enfermedad (Markowitz et al., 2012).

El objetivo de este trabajo es el análisis de las propiedades psicométricas de la versión española de la escala PAID-PR.

\section{MÉTODOS}

\section{Participantes}

En el estudio se recogieron los datos de 237 participantes, 146 madres y 91 padres con una edad media de $43.78( \pm 6.14)$ años. Los progenitores, entre los que había 75 parejas, eran asistentes a talleres psicoeducativos sobre diabetes. La edad de sus hijos/as oscilaba entre los 2 y 17 años $(\mathrm{M}=10.66 \pm 3.81)$ y la duración media de su diabetes tipo 1 fue de $3.86( \pm 2.8)$ años. El estudio fue aprobado por la comisión ética de la institución y los participantes firmaron el consentimiento informado.

\section{Medidas}

Escala de Áreas Problemáticas en la Diabetes para Padres-Revisada (PAID-PR; Markowitz et al., 2012). La PAID-PR original contiene 18 ítems (escala Likert de 5 
puntos; 0 = de acuerdo a 4 = en desacuerdo), disponibles en Markowitz et al., (2012), que describen aspectos de la diabetes que pueden preocupar a padres y madres (p.ej. “Me preocupo por el futuro y la posibilidad de que mi hijo/a tenga complicaciones graves", "Siento que la diabetes me consume demasiada energía física y mental"). Puntuaciones más elevadas indican niveles mayores de estrés. La versión española de la PAID-PR se elaboró mediante un proceso de traducción directa e inversa por traductores independientes (Bradley, 1994). Ya que algunos ítems son adaptaciones de la escala para adultos, la traducción directa de estos ítems se comparó con sus correspondientes en la versión española PAID ya validada en adultos. Volvió a traducirse al inglés y esta versión se comparó con la original PAID-PR para identificar posibles inconsistencias.

Escala de Ansiedad Rasgo (STAI-R; Spielberger, Gorsuch, \& Lushene, 1982). Se compone de 20 ítems (escala Likert de 4 puntos) que se refieren a los componentes cognitivos y somáticos de la ansiedad como rasgo de personalidad. La consistencia interna de la escala fue de .91 para la muestra de madres y de .88 en la de padres.

\section{Análisis de datos}

Debido a que en el estudio original no aparece el porcentaje de varianza explicado por la solución factorial alcanzada y se desconoce si algún ítem carga en más de un factor, se optó por una estrategia exploratoria de análisis (AFE). Así, con el objetivo de examinar la estructura subyacente a la versión española de la PAID-PR se realizó un análisis factorial exploratorio de ejes principales con rotación promax con las puntuaciones de los padres y de las madres separadamente. Para determinar el número de factores se usó el análisis paralelo (AP) mediante la aplicación desarrollada por Patil, Surendra, Mishra, \& Donovan (2008) disponible en (http://ires.ku.edu/ smishra/parallelengine.htm) que calcula autovalores medios 
extraídos de matrices de correlación generadas aleatoriamente con los que comparar los autovalores reales estimados. La consistencia interna de la escala se calculó mediante el coeficiente alfa de Cronbach. Respecto a la validez concurrente, se calcularon los coeficientes de correlación de Pearson entre las puntuaciones en la PAID-PR con las de ansiedad-rasgo, edad y duración de la diabetes. Se espera que el rasgo ansiedad, que implica una propensión a experimentar ansiedad en un amplio espectro de situaciones, se relacione con el estrés asociado al cuidado de hijos/as con diabetes. Para analizar las diferencias en las puntuaciones de la PAID-PR en función del género se realizaron contrastes de medias mediante pruebas T. El análisis de datos se llevó a cabo mediante el programa IBM SPSS 24.0.

\section{RESULTADOS}

Aunque los AFE con las puntuaciones de las madres $(\mathrm{KMO}=0.90)$ y de los padres en la PAID-PR $(\mathrm{KMO}=0.87)$ resultaron en una estructura inicial de cuatro factores con autovalores $\geq 1$ (madres: 7.74, 1.58, 1.30, 1; padres: 7.34, 2.06, 1.22, 1.07), el análisis paralelo indicó que deberían extraerse dos factores. Se eliminó el ítem 1 al presentar una carga menor de .35 en ambas muestras. El primer factor (PAID-PR I) agrupó 10 ítems (varianza explicada: madres, $41.43 \%$; padres, $38.87 \%$ ) referidos a las reacciones emocionales y preocupaciones sobre la diabetes y su cuidado (p.ej.: "me preocupo por si mi hijo/a tiene bajones de azúcar"). El segundo factor (PAID-PR II) incluyó 7 ítems (varianza explicada: madres, $6.48 \%$; padres, $9.53 \%$ ) relativos a la sobrecarga y la falta de apoyo en el cuidado de la diabetes (p.ej.: "me siento quemado/a por el esfuerzo constante que supone el manejo de la diabetes"). Las correlaciones entre los factores fueron de .67 y $.56(\mathrm{p}<.001)$ para madres y padres, respectivamente. La Tabla 1 incluye los estadísticos descriptivos de los ítems, las correlaciones ítem-total y las cargas factoriales tras la rotación, separadamente para madres y padres. Los coeficientes 
de fiabilidad de los factores extraídos fueron adecuados (ver Tabla 2). Respecto a la validez concurrente, los niveles de estrés se asociaron positivamente con las puntuaciones en el rasgo ansiedad. Ni la edad de los hijos/as ni la duración de la diabetes se relacionaron con los niveles de estrés. Asimismo, las madres informaron de mayores niveles de estrés que los padres (ver Tabla 2).

\section{DISCUSIÓN}

El objetivo de este estudio era examinar las propiedades psicométricas de la versión española de la escala PAID-PR. Al igual que en el estudio original (Markowitz et al., 2012), en nuestro análisis emerge una estructura de dos factores. Aunque entre ambas estructuras existen coincidencias en algunos de los ítems de cada factor ( 7 ítems del factor 1 y 5 ítems del factor II), aparecen diferencias en el número de ítems por factor y en su interpretabilidad y denominación. La versión española muestra una estructura claramente interpretable. En el primer factor se agrupan ítems que aluden tanto a reacciones emocionales como a preocupaciones por el cuidado y la adaptación de sus hijos/as. El segundo factor reúne ítems que se refieren a la sobrecarga o agotamiento del cuidado junto con otros relativos a la percepción de falta de apoyo para afrontar esa demanda excesiva. Por otra parte, el patrón de relaciones entre la PAID-PR y las medidas relacionadas con el estrés y con la diabetes representan un soporte empírico para la validez de la escala. Además, las diferencias en estrés en función del género están en la línea de la investigación previa (Haugstvedt et al., 2011). Las madres, que suelen asumir una mayor carga del cuidado de sus hijos/as, muestran niveles de estrés más altos. Por último, aunque este estudio incluye una proporción considerable de padres, a diferencia de estudios previos en los que son minoría (Hessler et al., 2016), la menor proporción de padres podría explicar los problemas con algunos ítems. Por último, este trabajo presenta algunas limitaciones. No se han analizado todos los 
aspectos de la validez de la escala. Se deberían examinar las relaciones de la PAID-PR con otros aspectos específicos de la diabetes como el nivel de conflictos asociados al reparto de responsabilidades en las tareas de cuidado, especialmente en progenitores de adolescentes. Además, aunque la diferencia de estrés por género se explicaría por la mayor carga de las madres, debería medirse esta carga evaluando la distribución de tareas de cuidado entre madres y padres. Futuros análisis factoriales confirmatorios con muestras más amplias permitirán corroborar la estructura factorial y su equivalencia entre madres y padres.

En conclusión, nuestros resultados sugieren que la escala PAID-PR constituye una medida breve, fiable y válida para la evaluación del estrés específico en madres y padres de niños/as y adolescentes con diabetes de utilidad en el ámbito clínico y de investigación.

\section{Agradecimientos}

La autora agradece el apoyo de la Fundación para la Diabetes (Madrid) para realizar este trabajo y a los/as revisores/as que proporcionaron útiles sugerencias a la versión previa de este trabajo.

\section{Referencias bibliográficas}

Barreiro, S. C., Rigual, M. R., Lozano, G. B., Siguero, J. L., Pelegrín, B. G., Val, M. R., \& Dea, M. C. (2014). Epidemiología de la diabetes mellitus tipo 1 en menores de 15 años en España. Anales de Pediatría, 81(3), 189.e1-189.e12.

Beléndez, M., Hernández-Mijares, A., Marco, J., Domínguez, J. R., \& Pomares, F. J. (2014). Validation of the Spanish version of the Problem Areas in Diabetes (PAID-SP) Scale. Diabetes Research and Clinical Practice, 106(3), e93-e95. 
Bradley, C. (1994). Translation of questionnaires for use in different languages and cultures. En C. Bradley (Ed.), Handbook of Psychology and Diabetes (pp. 4355). Reading: Harwood Academic Publishers.

Cousino, M. K., \& Hazen, R. A. (2013). Parenting stress among caregivers of children with chronic illness: a systematic review. Journal of Pediatric Psychology, $38(8), 809-828$.

Haugstvedt, A., Wentzel-Larsen, T., Rokne, B., \& Graue, M. (2011). Perceived family burden and emotional distress: similarities and differences between mothers and fathers of children with type 1 diabetes in a population-based study. Pediatric Diabetes, 12(2), 107-114.

Hessler, D., Fisher, L., Polonsky, W., \& Johnson, N. (2016). Understanding the areas and correlates of diabetes-related distress in parents of teens with type 1 diabetes. Journal of Pediatric Psychology, 41(7), 750-758.

Markowitz, J. T., Volkening, L. K., Butler, D. A., Antisdel-Lomaglio, J., Anderson, B. J., \& Laffel, L. M. B. (2012). Re-examining a measure of diabetes-related burden in parents of young people with Type 1 diabetes: the Problem Areas in Diabetes Survey-Parent Revised version (PAID-PR). Diabetic Medicine, 29(4), 526-530.

Nieuwesteeg, A., Hartman, E., Emons, W., Bakel, H., Aanstoot, H. J., Mil, E., \& Pouwer, F. (2017). Paediatric parenting stress in fathers and mothers of young children with Type 1 diabetes: a longitudinal study. Diabetic Medicine, 34(6), $821-827$

Patil, V.H., Surendra, N. S., Mishra, S., \& Donovan, T. Parallel analysis engine to aid determining number of factors to retain [Computer software]. Utilidad desarrollada como parte de Patil, V.H., Surendra, N. S., Mishra, S., \& Donovan, 
T. (2008). Efficient theory development and factor retention criteria: A case for abandoning the 'Eigenvalue Greater Than One' criterion. Journal of Business Research. 2007, 61(2), 162-170.

Pinquart, M. (2018). Parenting stress in caregivers of children with chronic physical condition-A meta-analysis. Stress and Health, 34,197-207.

Polonsky, W. H., Anderson, B. J., Lohrer, P. A., Welch, G., Jacobson, A. M., Aponte, J. E., \& Schwartz, C. E. (1995). Assessment of diabetes-related distress. Diabetes Care, 18(6), 754-760.

Snoek, F. J., Pouwer, F., Welch, G. W., \& Polonsky, W. H. (2000). Diabetes-related emotional distress in Dutch and US diabetic patients: cross-cultural validity of the problem areas in diabetes scale. Diabetes Care, 23(9), 1305-1309.

Spielberger, C. D., Gorsuch, R., \& Lushene, R. E. (1982). Cuestionario de ansiedad Estado-Rasgo. Autoevaluación. Madrid: TEA Ediciones.

Whittemore, R., Jaser, S., Chao, A., Jang, M., \& Grey, M. (2012). Psychological experience of parents of children with type 1 diabetes: a systematic mixedstudies review. The Diabetes Educator, 38(4), 562-579. 
Tabla 1. Estadísticos descriptivos, correlaciones item-total y cargas factoriales rotadas (matriz patrón) separadamente para madres/padres.

\begin{tabular}{|c|c|c|c|c|c|c|c|c|}
\hline & & Madres & & & & Padres & & \\
\hline Items & $\mathrm{M} \pm \mathrm{DT}$ & $\begin{array}{c}\text { Corr } \\
\text { Item-total }\end{array}$ & $\begin{array}{c}\text { PAID-PR } \\
\text { I }\end{array}$ & $\begin{array}{c}\text { PAID-PR } \\
\text { II }\end{array}$ & $\mathrm{M} \pm \mathrm{DT}$ & $\begin{array}{c}\text { Corr } \\
\text { Item-total }\end{array}$ & $\begin{array}{c}\text { PAID- } \\
\text { PR I }\end{array}$ & $\begin{array}{c}\text { PAID-PR } \\
\text { II }\end{array}$ \\
\hline 7 & $2.71 \pm .94$ & .67 & .79 & -.04 & $2.50 \pm .99$ & .63 & .78 & -.06 \\
\hline 10 & $2.93 \pm 1.01$ & .57 & .79 & -.16 & $2.75 \pm 1.02$ & .66 & .75 & .00 \\
\hline 11 & $3.04 \pm .95$ & .60 & .74 & -.06 & $2.65 \pm .92$ & .66 & .49 & .29 \\
\hline 6 & $2.99 \pm .89$ & .61 & .74 & -.05 & $2.57 \pm .87$ & .60 & .72 & -.02 \\
\hline 2 & $2.39 \pm 1.07$ & .65 & .72 & -.00 & $1.93 \pm 1.09$ & .64 & .70 & .04 \\
\hline 8 & $2.15 \pm 1.25$ & .61 & .57 & .11 & $1.73 \pm 1.20$ & .59 & .63 & .06 \\
\hline 4 & $1.95 \pm 1.03$ & .55 & .56 & .04 & $1.79 \pm 1.15$ & .50 & .56 & .00 \\
\hline 5 & $1.94 \pm 1.29$ & .53 & .54 & .04 & $1.70 \pm 1.38$ & .61 & .85 & -.15 \\
\hline 12 & $2.70 \pm 1.04$ & .61 & .52 & .16 & $2.23 \pm 1.07$ & .53 & .51 & .10 \\
\hline 3 & $1.60 \pm 1.29$ & .57 & .51 & .13 & $1.37 \pm 1.17$ & .54 & .50 & .10 \\
\hline 15 & $1.50 \pm 1.36$ & .59 & -.17 & .91 & $.80 \pm 1.03$ & .57 & -.04 & .75 \\
\hline 14 & $2.16 \pm 1.22$ & .68 & .10 & .71 & $1.35 \pm 1.07$ & .61 & -.11 & .91 \\
\hline 18 & $1.84 \pm 1.33$ & .64 & .06 & .71 & $1.21 \pm 1.20$ & .60 & -.04 & .81 \\
\hline 16 & $1.02 \pm 1.28$ & .44 & -.15 & .68 & $.71 \pm 1.15$ & .42 & -.00 & .53 \\
\hline 13 & $2.26 \pm 1.24$ & .69 & .28 & .52 & $1.76 \pm 1.14$ & .61 & .03 & .74 \\
\hline 17 & $1.97 \pm 1.10$ & .69 & .34 & .45 & $1.48 \pm .92$ & .57 & .20 & .48 \\
\hline 9 & $1.95 \pm 1.20$ & .59 & .30 & .37 & $1.65 \pm .95$ & .58 & .22 & .49 \\
\hline
\end{tabular}


Tabla 2. Puntuaciones medias del PAID-PR (totales y por factores), fiabilidad y correlaciones bivariadas con edad, duración de la diabetes y rasgo ansiedad separadamente para madres/padres.

\begin{tabular}{|c|c|c|c|c|c|c|c|}
\hline & $\begin{array}{l}\text { Rango } \\
\text { teórico }\end{array}$ & $\begin{array}{c}\text { Madres } \\
(\mathrm{N}=146) \\
\mathrm{M} \pm \mathrm{DT}\end{array}$ & $\begin{array}{c}\text { Padres } \\
(\mathrm{N}=91) \\
\mathrm{M} \pm \mathrm{DT}\end{array}$ & $\begin{array}{c}\alpha \\
\text { Cronbach }\end{array}$ & $\begin{array}{l}\text { Edad } \\
\text { hijo/a }\end{array}$ & $\begin{array}{c}\text { Duración } \\
\text { diabetes }\end{array}$ & STAI-R \\
\hline 1. PAID-PR-TOTAL & $0-100$ & $54.66 \pm 19.06^{\mathrm{a}}$ & $44.51 \pm 17.39^{\mathrm{a}}$ & $.92 / .91$ & $-.05 /-.18$ & $-.06 /-.03$ & $.61 * * / .58 * *$ \\
\hline 2. PAID-PR-I & $0-100$ & $61.11 \pm 18.99^{\mathrm{a}}$ & $53.17 \pm 19.37^{\mathrm{a}}$ & $.88 / .89$ & $-.11 /-.13$ & $-.06 /-.06$ & $.52 * * / .52 * *$ \\
\hline 3. PAID-PR-II & $0-100$ & $45.44 \pm 23.50^{\mathrm{a}}$ & $32.14 \pm 20.03^{\mathrm{a}}$ & $.87 / .86$ & $.02 /-.21 *$ & $-.06 /-.02$ & $.61 * * / .50 * *$ \\
\hline
\end{tabular}

PAID-PR-T (17 ítems): Estrés total; Rango teórico: posible puntuación normalizada (media x 25).

$\mathrm{M}=$ media, DT $=$ desviación típica. ${ }^{\text {a }}$ Medias significativamente diferentes en función del género $(p<.01)$

Coeficientes de fiabilidad de las escalas ( $\alpha$ Cronbach) y correlaciones entre variables (madres/padres)

${ }^{*} p<.05$. ${ }^{* *} p<.01$. 